\title{
Research and Implementation of OCPP 1.6 Protocol
}

\author{
Chaoyue Zhao ${ }^{1, a}$, Xiangdong You ${ }^{2, b, *}$ \\ ${ }^{1}$ Beijing University of Posts and Telecommunications, \\ School of Information and Telecommunication Engineering, No.10 Tucheng Road(West), Beijing, China \\ ${ }^{2}$ Beijing University of Posts and Telecommunications, \\ School of Information and Telecommunication Engineering, No.10 Tucheng Road(West), Beijing, \\ China \\ a18811396149@163.com, byouxiangdong@bupt.edu.cn
}

Keywords: New Energy, Electric Vehicle, Charge Point, OCPP 1.6.

\begin{abstract}
With the development of new energy vehicles in technology and industry, as well as the government's financial incentives and related policies' support, the new energy vehicles are constantly popularization and development, especially electric vehicles. More and more people choose electric vehicles as their travel tools. However, the charging facilities are imperfect, nonstandard and charging standards are not unified. There is no unified communication protocol between the various charging pile manufacturers, which makes the charging pile difficult to popularize. These factors restrict the development of new energy vehicles, especially electric vehicles. The emergence of OCPP 1.6 standardization provides a practical and effective solution for the integration and globalization of charging protocol.
\end{abstract}

\section{Introduction}

After the financial crisis, the world's major countries regard the development of electric vehicle industry as their national strategy, and the development of new industries becomes an important way to alleviate the energy crisis $^{[1]}$. China surpasses the United States and become the world's largest electric car country in production and sales in 2015. As a clean energy means of transport, electric vehicles has been more and more popular of all ages with its low fuel prices and security. However, the real restriction on the electric car is the construction of charging pile and the convenience of charging. The standardization and uniformization of the charging pile protocol, the interconnection communication between the equipments and the data communication between the charging pile manufacturers are the core problems to realize the rapid laying and use of the charging pile. The OCPP 1.6(Open Charging Protocol) proposed by the Open Charging Alliance (OCA) has been applied to more than 40, 000 charging facilities in 49 countries, it has become a global standard. This paper studied the OCPP 1.6 and simulated the transmission of the message specified by the protocol.

\section{About OCPP 1.6}

\subsection{What is OCPP (Open Charge Point Protocol)}

The Open Charging Alliance (OCA) is an international affiliate organization that including leading and business leaders in the public and private areas of electric vehicle infrastructure. The goal of the Open Charging Alliance is to promote the development and application of the charging protocol for electric vehicle charging facilities through cooperation, education, testing and certification, and to promote the industry standard of the relevant agreements. Open Charge Point Protocol (OCPP) is an open communication standard introduced by the Open Charging Alliance. It is mainly used to solve the difficulties of communication between private charging networks. OCPP supports communication between the Charge Point and the supplier's central management system. 
OCPP has been used in more than 40, 000 charging facilities in 49 countries, but at present, the State Grid, the Southern Power Grid, TELD, the Austrian Group and many other domestic charging equipment manufacturers and operators using the 104 agreement as the basis for their agreement. It is difficult to be in line with the international conventions.

\subsection{What is OCPP 1. 6}

OCPP 1.6 is based on OCPP 1.5. OCPP 1.5 has been widely used in the world since 2012, and many suppliers have applied the OCPP 1.5 standard in their products. These application experiences have been added to OCPP 1.6. A total of 19 companies have contributed their operating experience to OCPP1.6.

\subsection{The Differences between OCPP 1. 6 And OCPP 1.5}

OCPP 1.6 introduces new features to accommodate the market: Smart Charging, OCPP using JSON over Websockets, better diagnostics possibilities(Reason), more Charge Point Statuses and TriggerMessage. OCPP 1.6 is based on OCPP 1.5, with some new features and a lot of textual improvements, clarifications and fixes for all known ambiguities. Due to improvements and new features, OCPP 1.6 is not backward compatible with OCPP 1.5.

\subsection{Smart Charging Use Cases}

According to the OCPP 1.6, the charging system can be divided into two main parts, the Central System and the Charge Point. A Charge Point can have multiple connectors to connect with multiple electric vehicles. The main function of the protocol is to realize the information communication between the Central System and the Charge Point, so that the status and corresponding parameters of each transaction of the Charge Point are under the control of the Central System.

There may be many different uses for smart charging. The following three typical kinds of smart charging will be used to illustrate the possible behavior of smart charging ${ }^{[2]}$.

\subsubsection{Load Balancing Use Case Topology}

The Load Balancing use case is about internal load balancing within the Charge Point, the Charge Point controls the charging schedule per connector. The Charge Point is configured with a fixed limit, for example the maximum current of the connection to the grid.

The optional charging schedule field minChargingRate may be used by the Charge Point to optimize the power distribution between the connectors. The parameter informs the Charge Point that charging below minChargingRate is inefficient, giving the possibility to select another balancing strategy ${ }^{[2]}$.

The topology is shown in Figure 1.

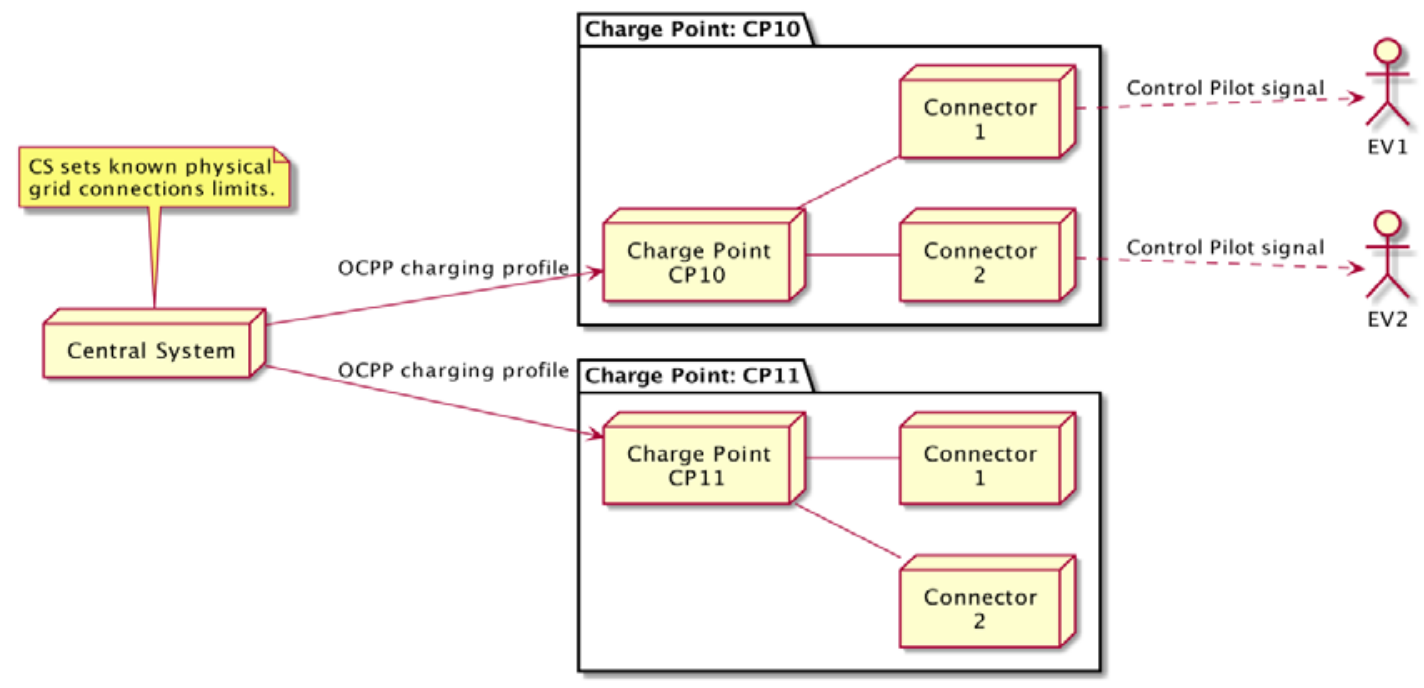

Figure 1 Load balancing Smart Charging topology. 


\subsubsection{Central Smart Charging}

With Central smart charging the constraints on the charging schedule, per transaction, are determined by the Central System. The Central System uses these schedules to stay within limits imposed by any external system. The Central System directly controls the limits on the connectors of the Charge Points ${ }^{[2]}$.

Central smart charging assumes that charge limits are controlled by the Central System. The Central System receives a capacity forecast from the grid operator(DSO) or another source in one form or another and calculates charging schedules for some or all charging transactions, details of which are out of scope of this specification.

The topology is shown in Figure 2.

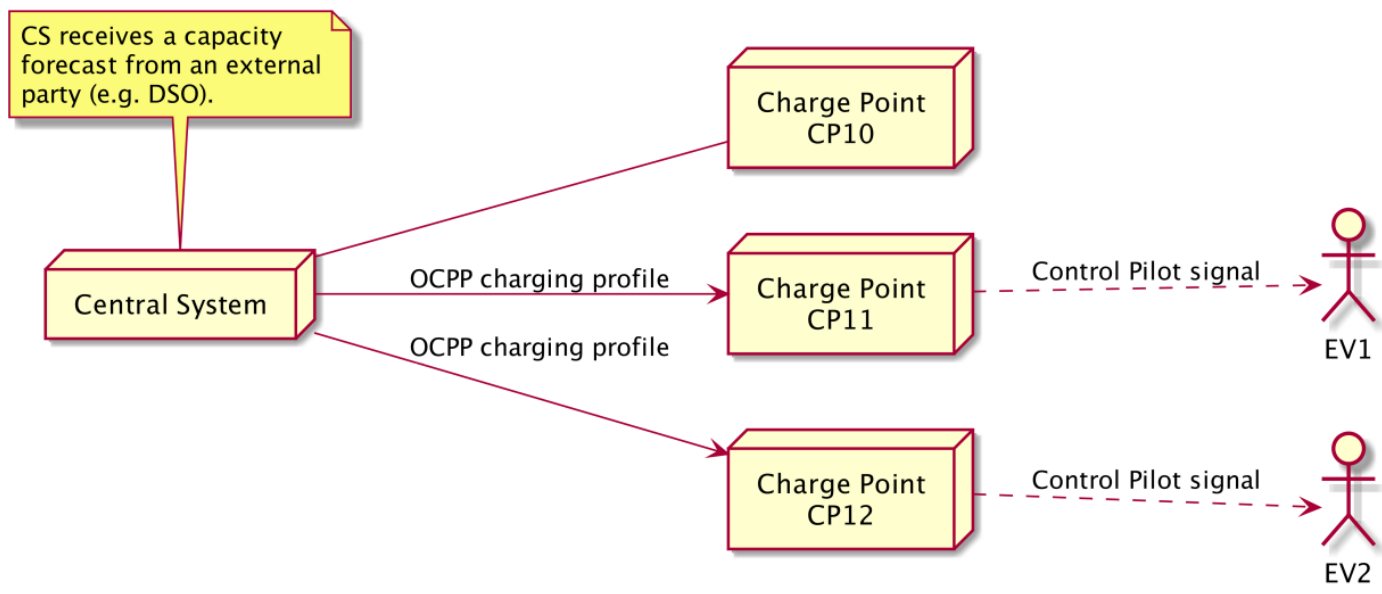

Figure 2 Central Smart Charging topology.

\subsubsection{Local Smart Charging}

The Local Smart Charging use case describes a use case in which smart charging enabled Charge Points have charging limits controlled locally by a Local Controller, not the Central System. The use case for local smart charging is about limiting the amount of power that can be used by a group of Charge Points, to a certain maximum. A typical use would be a number of Charge Points in a parking garage where the rating of the connection to the grid is less than the sum the ratings of the Charge Points. Another application might be that the Local Controller receives information about the availability of power from a DSO or a local smart grid node.

Local smart charging assumes the existence of a Local Controller to control a group of Charge Points. The Local Controller is a logical component. It may be implemented either as a separate physical component or as part of a 'master' Charge Point controlling a number of other Charge Points. The Local Control implements the OCPP protocol and is a proxy for the group members' OCPP messages, and mayor may not have any connectors of its own.

In the case of local smart charging the Local Controller imposes charging limits on a Charge Point. These limits may be changed dynamically during the charging process in order to keep the power consumption of the group of Charge Points within the group limits. The group limits may be preconfigured in the Local Controller or may have been configured by the Central System.

The topology is shown in Figure 3. 


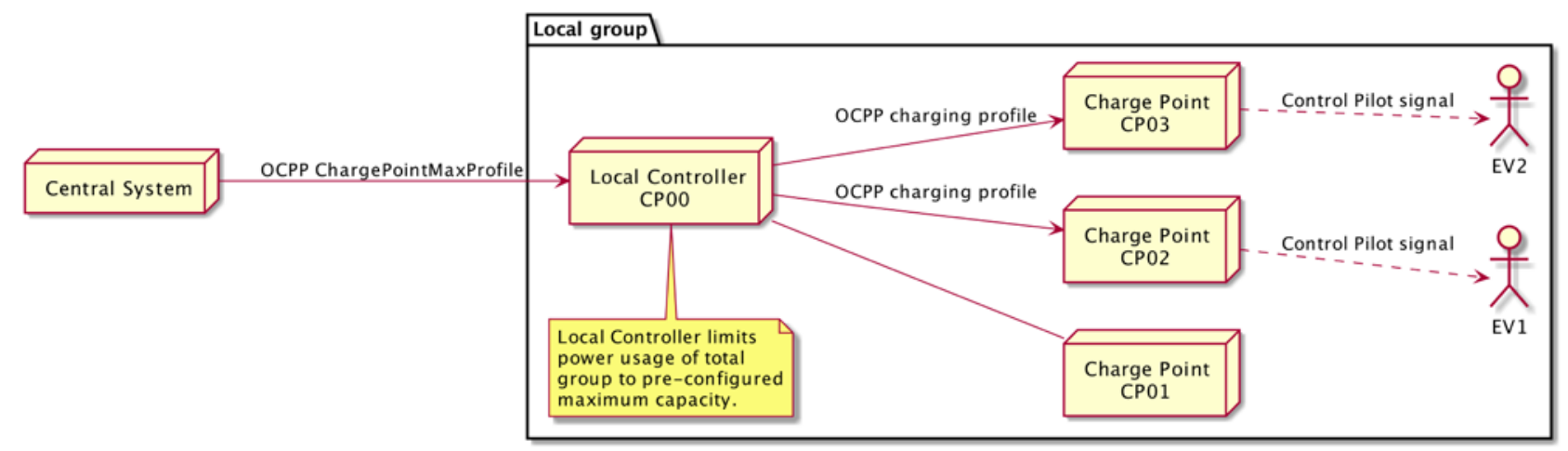

Figure 3 Local Smart Charging topology.

\subsection{Introduction to Related Transmission Parameters}

This part will introduce the transfer function and related parameters defined in the OCPP 1.6.

In OCPP 1.6, the Central System and the Charge Point need to transmit and receive messages to each other, that is, send the request messages and confirm messages. Since the transmission of messages is mutual, all messages can be divided into two types, that is, messages sent by the Central System and messages sent by the Charge Point ${ }^{[3]}$. The Central System sends a message to obtain the current state information or the control action of the Charge Point ${ }^{[4]}$. The message sent by the Charge Point is more about information about the beginning and end of the transaction and heartbeat, sample value and other data related to the business. The following are the Charge Point request and the Central System request.

\subsubsection{Charge Point Request: Heartbeat.req}

A Charge Point sends a heartbeat after a configurable time interval to let the Central System know that a Charge Point is still connected. The Charge Point will send a Heartbeat.req request, which does not contain any parameters. Upon receipt of this request, the Central System will return a Heartbeat.conf(currenttime) confirmation message indicating that the Central System has received a heartbeat request and has responded. This response carries a parameter named currenttime that indicates the time of receipt of the request and confirmation. It is a data of the DateTime type defined in the protocol. The transmission diagram is as Figure 4.

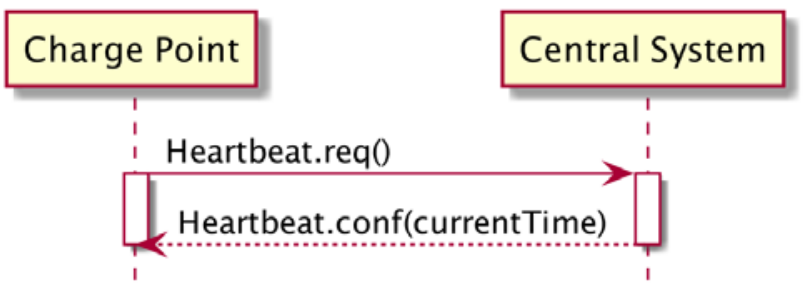

Figure 4 Sequence diagram:Heartbeat.

\subsubsection{Central System Request: ReserveNow.req}

A Central System can issue a ReserveNow.req to a Charge Point to reserve a connector for use by a specific idTag.

To request a reservation the Central System send a ReserveNow.req PDU(Protocol Data Unit) to a Charge Point. The Central System may specify a connector to be reserved. Upon receipt of a ReserveNow.req PDU, the Charge Point shall respond with a ReserveNow.conf PDU. It carries a parameter named status. The optional values are:Accepted, Faulted, Occupied, Rejected, Unavailable.The transmission diagram is as Figure 5. 


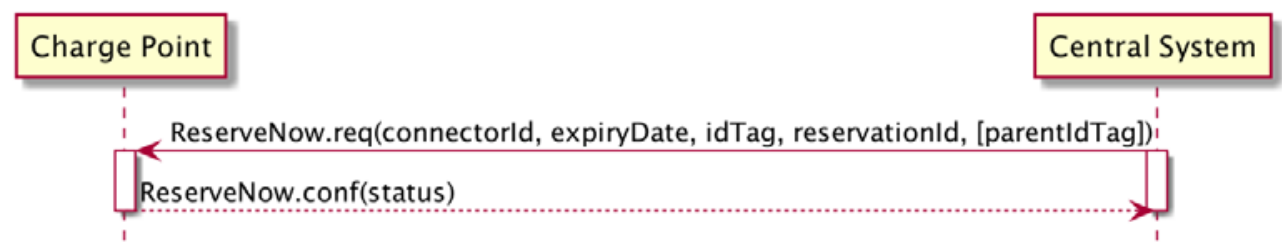

Figure 5 Sequence diagram: Reserve Now.

\section{The Technology Used To Implement OCPP 1.6}

Through the OCPP1.6 protocol reading and research, I set up a web server so that the Central System and Charge Point can communicate with each other.The key technologies used in this project are Jsp(dynamic web technology), MySQL, HTML+CSS technology, JavaScript technology and Maven(project management tool).

\section{Protocol Message Delivery Demonstration}

\subsection{Project Deployment}

First of all, this article refers to a specific implementation of a server on the Internet. A clientside code was written to implement the message transfer between the Charge Point and the Central System. The process of building a server is as follows.

- First download the project code from GitHub at the following address: https://github.com/RWTH-i5-IDSG/steve.

- Create a database named stevedb in the local database.

-In the steve-master folder, execute "shift+right mouse" to run the DOS command window, enter "mvn compile" to compil project.

-Enter "mvn package" to generate the target directory, compile and test the code and generate a test report.

- After all the commands are run, execute the following command in the DOS command window to execute the jar package:Java -jar target/steve-2.1.0.jar.

\subsection{Service Terminal Page}

\subsubsection{Login Page}

This page mainly implements the administrator's login so the administrator can monitor the Charge Point's information and state, and control the Central System to send messages to the Charge Point. The screenshot is as Figure 6.

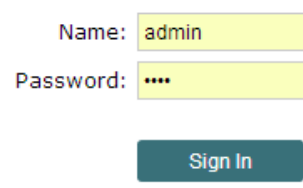

Figure 6 Login page.

\subsubsection{User Registration Page}

The user registration page is used to register information about these users who can use the Charge Point. Once the users' information is registered, the Central System can activate the charging service by authorizing the authentication after the Charge Point initiates the authentication request to the Central System, and the authorized user can carry out the charging activities. The screenshot is as Figure 7. 


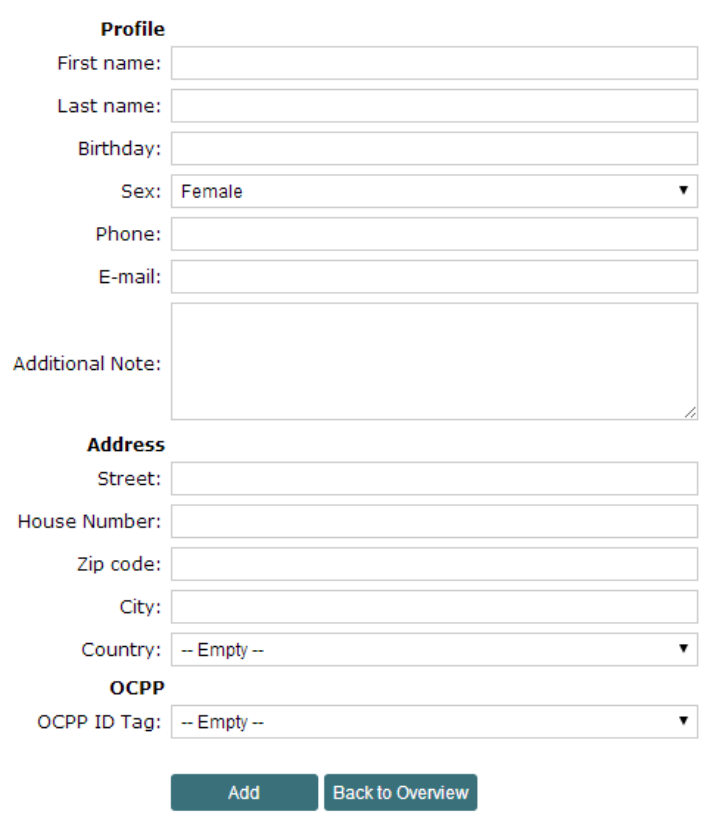

Figure 7 User registration page.

\subsubsection{Charge Point Registration Page}

The id of the Charge Point is the only and special identity of the Charge Point. This page completes the registration of the Charge Point and uniquely identifies its id so that the Central System can control the Charge Point accurately. This page also contains information such as Charge Point's location and etc. The screenshot is as Figure 8.

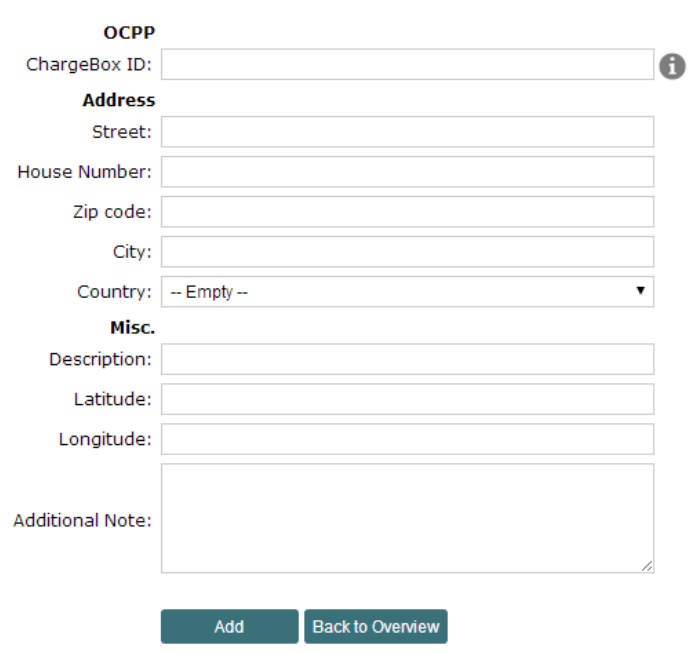

Figure 8 Charge Point registration page.

\subsubsection{Charge Point Overview Page}

The Charge Point overview page lists the charging information and the last heartbeat request time. The screenshot is as Figure 9.

\begin{tabular}{|c|c|c|c|c|}
\hline ChargeBox ID & Description & OCPP Protocol & Last Heartbeat & Add New \\
\hline no1 & 12 & & & Delete \\
\hline
\end{tabular}

Figure 9 Charge Point overview page. 


\subsubsection{Charge Point Status Information Overview Page}

This page intuitively lists the number of users, the number of charging points, the activities of the reserved business and the number of ongoing business, charging interfaces, heartbeats and other information. This page facilitates the overall control of the administrator. The screenshot is as Figure 10.

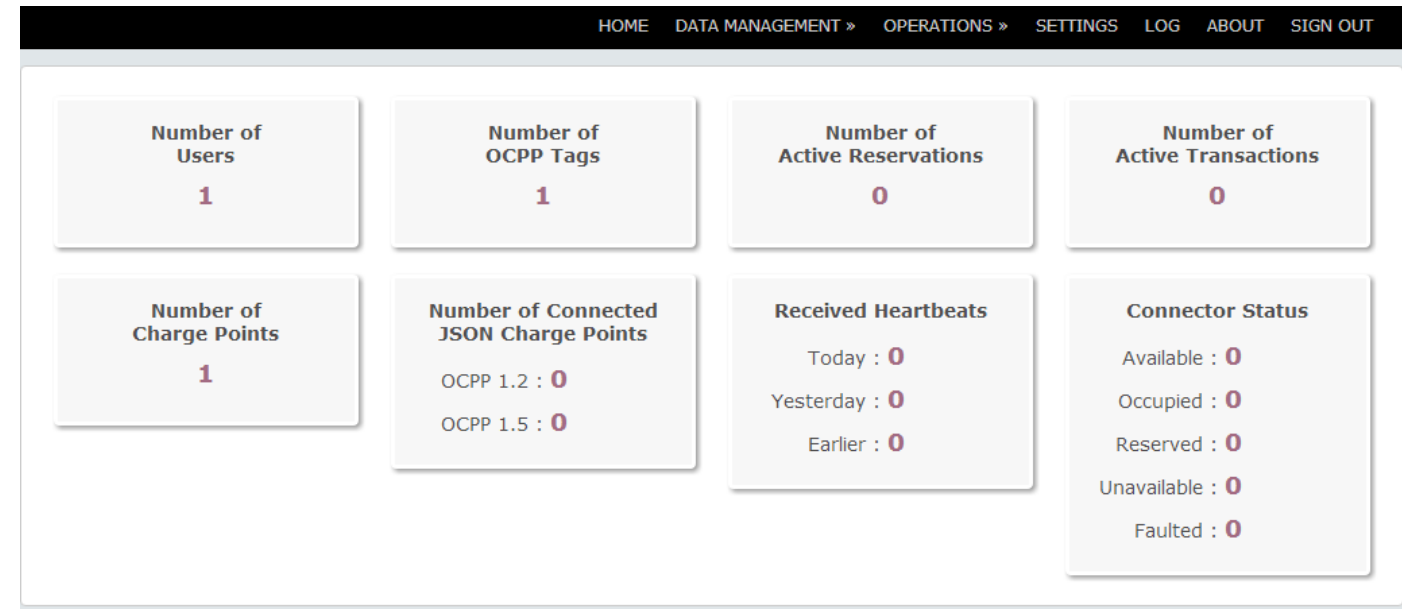

Figure 10 Charge Point status information overview page.

\subsection{Send Heartbeat Request}

The figure below shows the SoapUI client sending a heartbeat to the server and the server receiving the heartbeat. The port that server receives the message is:

http://localhost:8080/steve/services/CentralSystemServiceOCPP15.

The screenshot is as Figure 11.

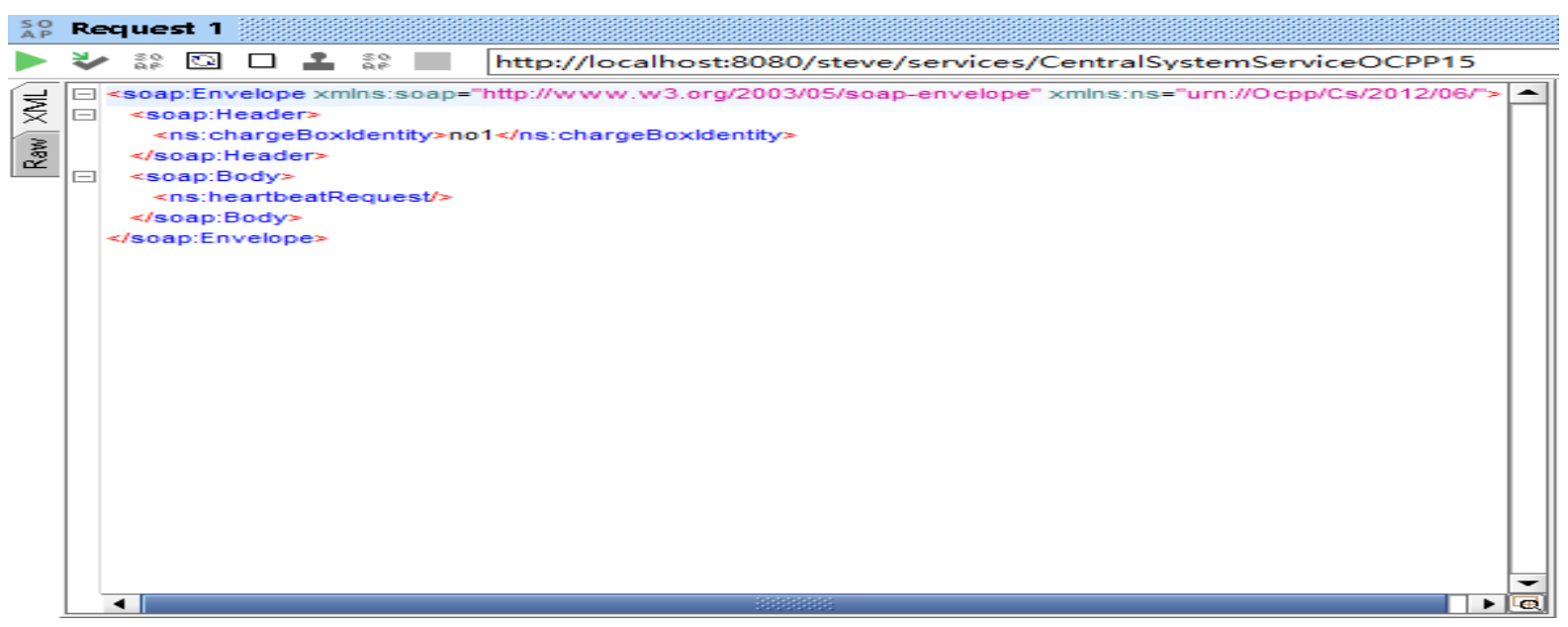

Figure 11 Send Heartbeat request.

After the soap message is sent successfully, the Central System will receive the message displayed in the Charge Point status page, as shown in the Figure 12.

\begin{tabular}{ccclc} 
ChargeBox ID & Description & OcPp Protocol & Last Heartbeat & Add New \\
\hline$\underline{\text { no1 }}$ & 12 & Today at 14:45 & Delete \\
\hline
\end{tabular}

Figure 12 Receive Heartbeat request.

\subsection{Send ReserveNow Request}

The sengding message page is shown as follow: 


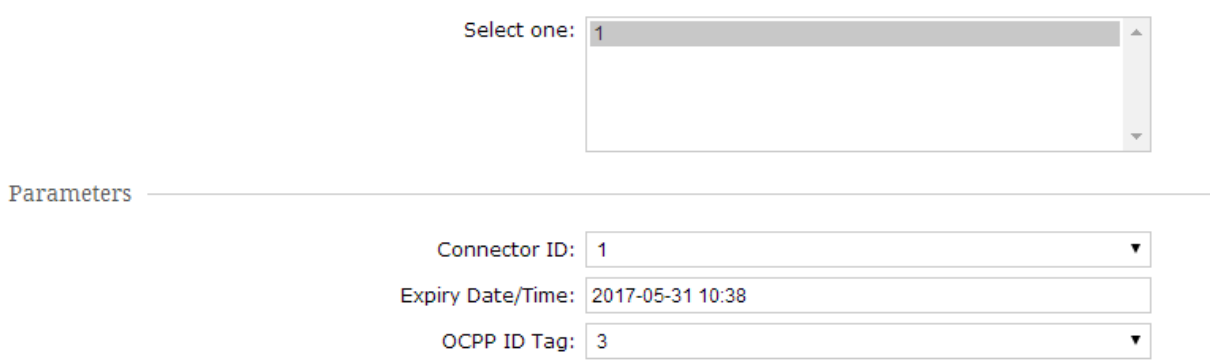

Figure 13 Send ReserveNow request.

The Central System wants to send a ReserveNow request to the No.1 connector on the No.1 Charge Point, with the expiration date set as Expire Date. When the ReserveNow request is not processed when the request exceeds the time limit, the ReserveNow request will expire.

When the message is sent, the message's status becomes waiting, waiting for the response message. The screenshot is as follow.

\begin{tabular}{cccccccc} 
Reservation ID & Transaction ID & OCPP ID Tag & ChargeBox ID & Connector ID & Start Date/Time & Expiry Date/Time & Status \\
\hline \hline 1 & $\underline{3}$ & $\underline{1}$ & 1 & Today at 02:50 & $2017-05-31$ at 10:38 & WAITING \\
\hline 2 & $\underline{3}$ & $\underline{1}$ & 1 & Today at 02:50 & $2017-05-31$ at 10:50 & WAITING \\
\hline 3 & $\underline{3}$ & $\underline{1}$ & 1 & Today at 03:02 & $2017-05-31$ at $11: 02$ & WAITING \\
\hline
\end{tabular}

Figure 14 ReserveNow request's status.

Once the ReserveNow request is accepted, the client returns a status field according to the status of the connector. The following figure indicats that the reservation was successful and a connector was reserved.

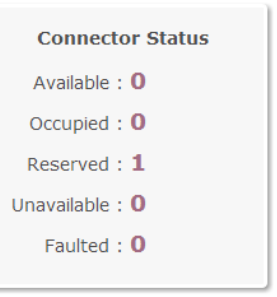

Figure 15 ReserveNow request sent successfully.

\section{Conclusions}

This paper introduces the role and function of the OCPP 1.6 and its effect on the development of the Charge Point. It also briefly describes the process of building the server and the key technologies needed. Then, based on the server, this paper implemented the message transmission defined by the protocol. Completed the expected objectives of this paper.

\section{Acknowledgements}

Firstly, I would like to express my sincere gratitude to my supervisor, XiangDong You, for his instructive advice and useful suggestions on my thesis. I am also indebted to all my friends for their direct and indirect help to me, special thanks should go to them for their comments on the project and the thesis.

\section{References}

[1] Cristina Alcaraz, Javier Lopez, Stephen Wolthusen (2017) OCPP Protocol: Security Threats and Challenges. IEEE Transactions on Smart Grid, 2, 1-1. 
[2] Open Charge Allianc (2015) Open Charge Point Protocol 1.6.

[3] Á.Rodríguez-Serrano, A.Torralba, E.Rodríguez-Valencia, J.Tarifa-Galisteo (2013) A communication system from EV to EV Service Provider based on OCPP over a wireless network. IEEE, 10, 5434-5438.

[4] Jens Schmutzler, Claus Amtrup Andersen, Christian Wietfeld (2013) Evaluation of OCPP and IEC 61850 for Smart Charging Electric Vehicles. Electric Vehicle Symposium and Exhibition, 10, $1-12$.

[5] T. Parker, D. Naberezhnykh (2013) Charging point strategies for electric commercial vehicles. Hybrid and Electric Vehicles Conference, 10, 1-4. 